\title{
Non-animal ultrasound phantoms for device testing and training
}

\section{F Passariello ${ }^{1}$}

${ }^{1}$ Fondazione Vasculab ONLUS, via Francesco Cilea 280 - 80127 Naples, Italy

presented to: Contribute to the Conference 'Sperimentazione non-animale. Un'alternativa possibile.' [Non animal experiments. A possible alternative.] Fondazione Humaniter. Napoli, Nov 29, 2017. submitted: Jun 27, 2018, accepted: Jun 29, 2018, EPub Ahead of Print: Jun 29, 2018, published: Jun 30, 2018 Conflict of interest: None

DOI: 10.24019/jtavr.26 - Corresponding author: Dr. Fausto Passariello, afunzionale@tiscalinet.it

(C) 2017 Fondazione Vasculab impresa sociale ONLUS. All rights reserved.

\begin{abstract}
A phantom is a physical object to simplify device testing and clinical training. An industrial device is more reliable for device testing in quality control procedures, though it is expensive. When precision requirements are not so strict as it occurs in training, people prefer to use home-made phantoms, though many of these artisanal methods use animal products. The current paper illustrates a few alternative vegetal phantoms. Agar is a widely used material in laboratory investigations and can be used to contain ultrasound targets. Another quick and effective alternative is given by tofu. Target sizes and flow ultrasound measurements can be easily effected using a phantom and training can be planned and repeated as much as required. The current paper shows how low-cost animal phantoms can be perfectly replaced by low-cost vegetal ones for clinical and training purposes. Vegetal phantoms can be classified as possible realizations of the Replace, the 1 st of the 3 Rs pre-requisites for non-animal experiments.
\end{abstract}

Keywords Ultrasound phantoms; agar phantoms; tofu phantoms; ultrasound training; 3Rs.

\section{Introduction}

Ultrasound imaging devices must be calibrated to be reliable measuring devices. For instance, the ultrasound probes and the internal procedures are projected to get the maximal resolution in the axial (depth) and transverse (lateral) directions.

Testing of imaging devices is accomplished using simplified models (phantoms), which contain several geometric objects of known size, to check the device capability of capturing the correct values. According to the use of the phantom, structures can be also traversed by a fluid, in order to simulate the behavior of a more complex but dynamic real condition.

Device testing requires a great precision in measurements as well as in the manufacturing of the phantoms, thus an industrial device is more reliable and preferable, because the aim is to perform a quality control test.

Several professional phantoms are on the market, generally provided by device manufacturers and made of synthetic materials. However, these phantoms are generally expensive and most people, especially in training, prefer to build their phantoms manually, for their personal and clinical environments as well as for an educational classcourse.

Many low-cost alternatives can be home-made, especially for training in diagnostic procedures or in ultrasound-guided surgical procedures. There is quite a lot of home-made instructions and movies on the web, which show how to get an easy and low-cost phantom. However, many of these artisanal methods use animal products like gelatin as medium to include geometrical ultrasound targets.

As an extreme case example, a simple method to train the emergency personnel in vascular access procedures, even without any ultrasound guide, uses a rubber tourniquet and a slice of turkey meat to simulate a vessel to get people 
acquainted with the needle puncture for vascular access ${ }^{1}$. Thus the usefulness of reviewing the already available methods to build a home-made vegetal phantom.

- $\quad$ nothing new is here presented, everything being already known, though generally not sufficiently well-known;

- $\quad$ the vegetal phantom shows similar properties in comparison with the other ones of animal origin;

- $\quad$ as regards precision and reliability, a homemade vegetal phantom cannot be compared at all to the synthetic industrial devices;

- $\quad$ the ready-made available devices are much more expensive.

\section{Vegetal phantoms}

\section{Agar phantoms}

Agar or agar-agar is a mixture of agarose and agaropectin. Agarose is a linear polysaccharide made by a linear sequence of two disaccharides ${ }^{2}$. Widely used in microbiology as a culture medium (Petri dish) and in general chemistry as a support for electrophoresis, its origin dates to the XVII century in Japan ${ }^{3}$.

Agar is insoluble in cold water, but it hydrates in boiling water, returning to a gel phase as soon as temperature is held below $35-45^{\circ} \mathrm{C}$. This property makes it easy and quick to get a support for an ultrasound phantom ${ }^{4,5}$. Placing several ultrasound targets (an orange, a nut, a straw or a tourniquet) into a plastic container and adding a warm solution of agar, it suffices to wait a while for its spontaneous cooling to get a stable gel phantom. The targets can be filled with still water or extraneous materials, which can generate acoustic shadows, according to their echogenicity, for instance simulating gallstones.

\section{Tofu phantoms}

Tofu, called also vegetal meat or bean curd, is the result of the coagulation of soy milk. Its origin dates to more than 2000 years ago in China, while the first use of the term in English dates to the XIX century in the USA6.

Tofu can be stored in different consistencies, but here an application is shown using an extra-firm tofu to build a phantom.

Take an extra-firm tofu slab and, profiting of its consistency, push inside it as many straws you want to simulate a vascular access. A tourniquet can be inserted in the straw and then the straw can be removed, to simulate a compressible vessel. Place all in a container and add cold water to cover the straws, but not the tofu surface. In such a way, water will fill the straws, leaving the surface dry instead.

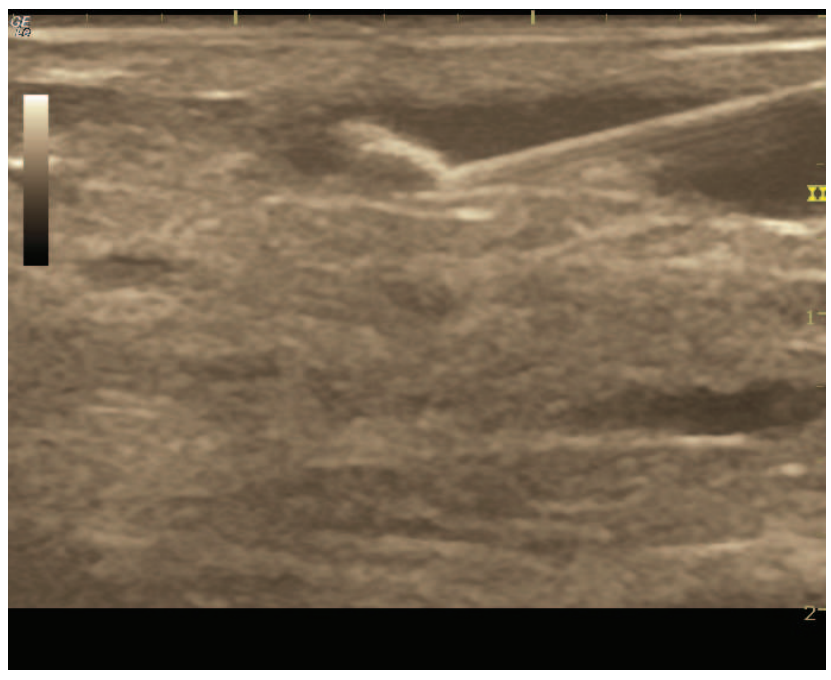

Figure 1 - A true vascular access for venous sclerotherapy in a human tributary vein of a lower limb. A thick jet of hyper-echogenic sclerosing foam exits the tip of the needle. Note the ultrasound artifact with repeated thin and light parallel images of the needle, is a general effect which can be also detectable with a phantom.

The described procedure is efficiently shown on a web movie ${ }^{7}$, where it is well emphasized how negligible is the required time to prepare the phantom. As the simulated vessels connect to the external container, any injection of material during the training procedure can exit to the outer container and the phantom is practically regenerated by simple washing and emptying of the container.

\section{Measurements and training}

Measuring the size of the ultrasound targets before including them into the container gives the possibility of training the ultrasound operators in the collection of reliable measures.

In addition, using a particle fluid and connecting the straws or tourniquets to a calibrated volumetric pump or reservoir allows the comparison of the flow measurements (change of levels in the reservoir or automatic pump calibration) with the ones made by ultrasound (Doppler velocity multiplied by the B-Mode ultrasound area).

As regards the vascular access, targets can be positioned at different depths in the phantom, in order to simulate difficulties which arise in deeper vessels, when the needle must be longer and the angle between the needle and the skin or the vessel is less acute.

Figure 1 show a true vascular access in the human, while figure 2 shows instead a simulated access on a tofu phantom. Having a phantom, you can bite as many times you want to learn the tricks of the chosen procedure. 


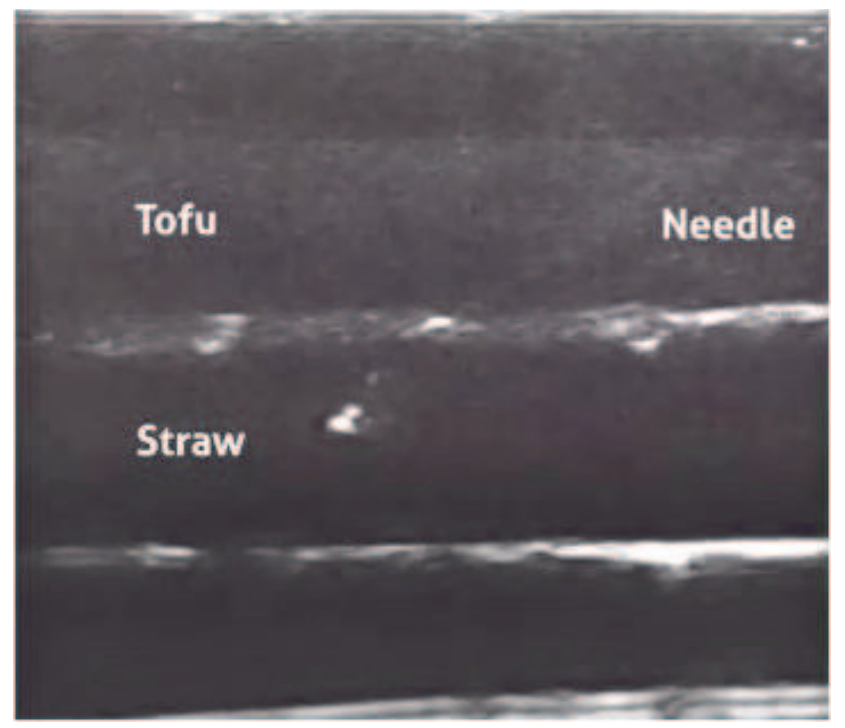

Figure 2 - A simulated vascular access on a tofu phantom. Taken from a Youtube educational movie (Eddie)

\section{References}

1) Passariello F. Sperimentazione non-animale. Un'alternativa possibile. [Non animal experi-ments. A possible alternative.] Conference at Fondazione Humaniter. Napoli, 2017, Nov 29.

2) Bin Luo, Ronghua Yang, Peng Ying, Awad M, Choti M, Taylor R. Elasticity and Echo-genicity Analysis of Agarose Phantoms Mimicking Liver Tumors. Proceedings of the IEEE 32nd Annual Northeast Bioengineering Conference [Internet]. IEEE; Available from: http://dx.doi.org/10.1109/nebc.2006.1629762

3) Zucca P, Fernandez-Lafuente R, Sanjust E. Agarose and Its Derivatives as Supports for En-zyme Immobilization. Molecules [Internet]. MDPI AG; 2016 Nov 19;21(11):1577. Available from: http://dx.doi.org/10.3390/molecules21111577

4) Mitchell MD, Kundel HL, Axel L, Joseph PM. Agarose as a tissue equivalent phantom ma-terial for NMR imaging. Magnetic Resonance Imaging [Internet]. Elsevier BV; 1986 Jan;4(3):263-6. Available from: http://dx.doi.org/10.1016/0730-725x(86)91068-4

\section{Conclusion}

For device testing, synthetic but expensive phantoms are already available to achieve reliable industrial laboratory results for quality control.

The current paper shows instead how low-cost animal phantoms can be perfectly replaced by low-cost vegetal ones for clinical and training purposes.

In addition, agar and tofu phantoms are of easy home-made manufacture from materials, which are widely available on the market. Their use is in agreement with the $1^{\text {st }}$ of the 3 Rs pre-requisites for non-animal experiments, i.e. Replace, Reduce, Refine, which are nowadays generally accepted $^{8}$.

5) Earle M, Portu GD, DeVos E. Agar ultrasound phantoms for low-cost training without re-frigeration. African Journal of Emergency Medicine [Internet]. Elsevier BV; 2016 Mar;6(1):18-23. Available from: http://dx.doi.org/10.1016/j.afjem.2015.09.003

6) Anonymous. Tofu. [Internet]. Last edited on 30 June 2018 [Accessed on Jun 30, 2018]. Available from: https://en.wikipedia.org/ wiki/Tofu

7) Eddie C. Easy DIY Tofu ultrasound phantom. YouTube movie. Available from:https://www.youtube.com/watch? $\mathrm{v}=\mathrm{utWZHA7gp-4.}$

8) European society for alternatives to animal testing. Available at the address http://www.eusaat-congress.eu/ at the date of Jun 30, 2018 